\title{
A new optical intra-tissue fiber irradiation ALA-PDT in the treatment of acne vulgaris in rabbit model: improved safety and tolerability ${ }^{*}$
}

\author{
Qian Wang ${ }^{1}$ \\ Wei Liu ${ }^{1}$ \\ Xinyu Lin $^{1}$ \\ Xiling Duan ${ }^{1}$
}

\author{
Can Jiang ${ }^{2}$ \\ Jin Chen ${ }^{1}$ \\ Xiangning Huang ${ }^{3}$
}

DOI: http://dx.doi.org/10.1590/abd1806-4841.20175543

\begin{abstract}
BACKGROUND: Photodynamic therapy with topical aminolevulinic acid (ALA-PDT) has been suggested to be effective in treatment of acne vulgaris. However, adverse events occur during and after treatment.

OвJестіves: To compare the efficacy and tolerability of optical intra-tissue fiber irradiation (OFI) ALA-PDT versus traditional ALA-PDT in treatment of acne vulgaris in rabbit models.

MetHods: Twenty-five rabbits of clean grade were used. Twenty rabbits were randomly selected to establish acne model and the other five were used as control. Rabbits in model group (40 ears) were further divided into four groups (10 ears/group): I, OFI-ALA-PDT with the head of optical fiber inserted into the target lesion (intra-tissue); II, traditional ALA-PDT group; III, OFI group; IV, blank control group without any treatment. Uncomfortable symptoms, adverse events, and effectiveness rates were recorded on post-treatment day 14,30 , and 45.

RESULTS: On post-treatment day 14, the effectiveness rate in OFI-ALA-PDT group was obviously higher than that of the other three groups $(\mathrm{P}<0.05)$. However, no improved effects were observed in OFI-ALA-PDT group on day 30 and 45 . During the period of treatment, the frequencies of uncomfortable symptoms in ALA-PDT group were obviously higher than those in the other three groups $(\mathrm{P}<0.05)$. The adverse event rate in OFI-ALA-PDT group was obviously lower than that of the ALA-PDT group $(\mathrm{P}<0.05)$. STUDY LIMITATIONS: The unblindness of the study and temporary animal models of acne induced may hamper the assessment and monitoring of the results, and future studies are still needed to clarify it further.

CONCLUSIONS: The OFI-ALA-PDT group (intra-tissue irradiation) showed no improved efficacy on treating rabbit ear acne but had higher safety and better tolerability.
\end{abstract}

Keywords: Acne Vulgaris; Safety; Treatment Outcome

\section{INTRODUCTION}

Acne vulgaris is a common skin disease affecting more than $80 \%$ of young people. Propionibacterium acnes and sebum secretion mainly contribute to the acne pathogenesis. The recommended treatments for acne comprise topical medications and systemic use of antibiotics, hormones, and retinoids according to severity. ${ }^{1}$ Due to an increasing antibiotic resistance of $P$. acnes and contraindications or adverse events of oral drugs, patients who cannot tolerate or are unwilling to accept drug treatment can choose physical therapy. At present, effective physical therapies include photodynamic therapy, laser treatment and fruit acid treatment.

Photodynamic therapy (PDT) with topical aminolevulinic acid (ALA) has been used for treatment of skin cancer, actinic keratoses, and psoriasis. Several studies have suggested that ALA-PDT is also effective in the treatment of acne vulgaris. ${ }^{1-5}$
However, adverse events occur in some patients both in the course of treatment (such as pain, burning sensation, itching, pricking, edema or erythema) and after the treatment (such as erythema, edema, pustules, acneiform eruption, exfoliative and secondary pigmentation). ${ }^{6-8}$ The reasons are related with the concentration of photosensitizer, energy of light, skin conditions of patients, and so on. But we cannot ignore that the whole face irradiation lack of accuracy is also an important reason.

Therefore, the aim of this study is to find a more subtle and minimally invasive form of irradiation. We attempted to improve the pathway of light, importing light directly into the target lesion with optical fiber to reduce the PDT adverse events by precise intra-lesional irradiation.

Received on 01.01.2016

Approved by the Advisory Board and accepted for publication on 03.05.2016

* Study conducted at the Institute of Dermatology and Venereology, Sichuan Academy of Medical Sciences \& Sichuan Provincial People's Hospital, Chengdu Sichuan, P. R. China.

Financial support: This study was supported by the Health and Family Planning Commission of Sichuan Province (150222).

Conflict of interest: None.

Institute of Dermatology and Venereology, Sichuan Academy of Medical Sciences \& Sichuan Provincial People's Hospital - Chengdu, China.

School of Clinical Medicine, Southwest Medical University - Luzhou, China.

Clinical Laboratory Department, Sichuan Academy of Medical Sciences \& Sichuan Provincial People's Hospital - Chengdu, China.

C2017 by Anais Brasileiros de Dermatologia 


\section{METHODS}

\section{Animal models}

This study has been approved by the ethics committee of the Sichuan Academy of Medical Sciences \& Sichuan Provincial People's Hospital. Twenty-five rabbits, 12 weeks old, with a mean body weight of about $2.0 \mathrm{~kg}$, were used in our study (provided by experimental animal center of Sichuan Provincial People's Hospital).

Establishment of the rabbit ear acne model

Twenty rabbits were randomly selected to establish acne model with the inoculation of $P$. acnes.${ }^{9-10} \mathrm{We}$ first coated $0.5 \mathrm{~mL}$ of crude coal tar everyday on each rabbit ear near the ear tube with an opening range of $2 \mathrm{~cm} \times 2 \mathrm{~cm}$ for two weeks. P. acnes liquid of $50 \mathrm{ul} /$ ear was inoculated by intradermal injection on day 7 . The remaining five rabbits were classified as normal control group. Visual inspection was performed seven days after injection, and five rabbits were randomly selected to undergo skin biopsy at the target acne lesion and the five ears of five rabbits in normal control group also underwent biopsy. All specimens were fixed in $10 \%$ formaldehyde solution, embedded in paraffin and cut into continuous six slices, then H\&E stain and examination under a microscope were conducted.

\section{Treatment}

The 20 model rabbits were randomly divided into four groups, with five rabbits (10 ears) in each group. Inflammatory papules with a diameter of more than $3 \mathrm{~mm}$ in each ear were selected. Rabbits were not on medication when under PDT treatment. The details are as followed:

Optical intra-tissue fiber irradiation ALA-PDT group (OFIALA-PDT): double layer of gauze soaked with 50\% ALA solution was applied to the rabbit ears and the ALA-applied area was occluded with plastic films. Gauze and plastic films were removed after two hours of incubation in the dark. The head of optical fiber $(0.2$ mm diameter) was then inserted into the center of the target lesion with a depth of approximately $2 \mathrm{~mm}$ (intra-tissue), importing the red light directly into the lesion by the red light emission spectrometer (designed by our group and manufactured by Shaanxi Richeng Technology Development Co.,Ltd., China). The wavelength of red light was $630 \mathrm{~nm}$, power density into the skin was $5 \mathrm{~mW} / \mathrm{cm}^{2}$, irradiation time was $15 \mathrm{~min}$.

Traditional ALA-PDT group (ALA-PDT): ALA incubation and red light irradiation dose was described like OFI-ALA-PDT group but the traditional external irradiation (irradiating for the whole ear region) (Wuhan Guangfu Bioengineering Co., Ltd., China) was used.

Optical fiber irradiation group (OFI): No photosensitizer was used. The head of optical fiber was directly inserted into the center of the target lesion with a depth of about $2 \mathrm{~mm}$. Treatment parameters were the same of OFI-ALA-PDT group.

Blank control group: This group received no treatment.

\section{Evaluation}

The rabbit ears were examined carefully and the numbers of inflammatory papules were counted and recorded at baseline and after 14, 30, and 45 days of treatment (these observation time points were chosen similar to the experimental used by humans). We used a rabbit ear as a unit to calculate the effectiveness rate. The clearance rate of lesions $(\%)=[($ the total number of skin lesions before treat- ment - the total number of skin lesions after treatment) / the total number of skin lesions before treatment] $\times 100 \%$. Therapeutic assessment was conducted as follows: cure, $\geq 90 \%$ clearance; excellent response, $60 \sim 89 \%$ clearance; good response, $20 \sim 59 \%$ clearance; poor response, $<19 \%$ clearance or no significant response. ${ }^{11}$

The effectiveness rate $(\%)=[$ (cure cases + excellent response cases) / total cases] $\times 100 \%$.

Adverse events occurred during the period of treatment: frequencies of uncomfortable symptoms such as throwing ears, twisting the body, digging with claws and screaming were recorded.

Adverse events occurred after treatment: adverse events of the skin after treatment, such as erythema, edema, pustule, desquamation, etc., were observed. The cases of rabbit ears that suffered adverse events were recorded and the incidence rate was calculated for each group. Incidence rate $=$ (number of cases with adverse events / total numbers of rabbit ears for each group) $\times 100 \%$.

\section{Statistical Analysis}

Data were analyzed using the SPSS 17.0 software. Measurement data were analyzed with $t$ test. For enumeration count data, Chi-square test was used. $P<0.05$ was considered statistically significant.

\section{RESULTS}

\section{The results of modeling}

Compared with the normal rabbit ears, the acne model ears manifested as comedones and inflammatory papules in naked eyes (Figure 1). Histological observation showed thickening of the epidermis, hyperkeratosis, expanded follicular with increased and dense keratosis, enlarged sebaceous glands, and infiltration of inflammatory cells in the dermis (Figure 1). All these manifestations were similar to human acne. In addition, mean diameter of sebaceous glands was all significantly greater than those in normal control group $(P<0.05$, Table 1$)$. Rabbit ears were thicker after establishing the model. These manifestations suggested that the rabbit ear model of acne was successfully established.

\section{Macroscopic observation}

After different treatment methods, three treatment groups obtained certain effects. The blank control group also self-healed gradually because there was no continuous artificial stimulation. The clinical outcomes at different time point are showed in Table 2 and Figure 2. At day 14, the effect difference was the clearest. But on day 30 and 45, no improved effects were observed in the OFI-ALAPDT group (Figure 2).

\section{Adverse events}

Adverse events occurred at the process of treatment: At the 15 min of treatment, every rabbit in the three treatment groups had experienced uncomfortable symptoms, like throwing ears, twisting the body, digging with claws and screaming. The frequency of discomfort, from most to least common, was as follows: ALA-PDT group (126 times) > OFI-ALA-PDT group (47 times) > OFI group (37 times). We can conclude that the occurrence of uncomfortable symptoms was much more frequent in traditional ALA-PDT group.

Adverse events occurred after treatment: OFI-ALA-PDT group: One rabbit ear had erythema around the target lesion after one day of irradiation (Figure 3). ALA-PDT group: four rabbit ears 
had erythema around the target lesions after one day of irradiation, one ear had pustules after two days of irradiation, one had rhagades after seven days of irradiation, two had hypopigmentation after 45 days of irradiation (Figure 3). OFI group: no ears had local adverse events. Table 3 shows the incidence rates of adverse events in different groups.

TABLE 1: Comparison of the mean diameter of sebaceous glands, the mean area of hair follicles between model group and normal control group

\begin{tabular}{lll} 
Group & No. & $\begin{array}{l}\text { Mean diameter of sebaceous } \\
\text { glands }(\boldsymbol{\mu m})\end{array}$ \\
\hline Normal control group & 10 & $694.967 \pm 184.845$ \\
Model group & 10 & $1555.912 \pm 612.369$ \\
T & & -5.562 \\
P-value & 0.000
\end{tabular}

Efficacy in different groups at different time point

\section{DISCUSSION}

Photodynamic therapy (PDT) is a therapeutic method used with increasing frequency in dermatology. ALA-PDT has been used in dermatology mainly for a cohort of proliferative, angiogenic, or inflammatory skin diseases such as cutaneous cancers, precancerous lesions, fungal infections, and HPV viral infections. ${ }^{12-15}$

TABLE 3: The incidence of adverse events in different treatment groups

\begin{tabular}{lllll} 
Group & No. of ears & \multicolumn{2}{c}{ Adverse event } & $\begin{array}{l}\text { Incidence } \\
\text { rate }(\%)\end{array}$ \\
& \multicolumn{5}{c}{ Yes } & No & \\
\hline OFI-ALA-PDT & 10 & 2 & 8 & $20^{*} \boldsymbol{\Delta}$ \\
ALA-PDT & 10 & 9 & 1 & $90^{*}$ \\
OFI & 10 & 1 & 9 & $10^{*} \boldsymbol{\Delta}$
\end{tabular}

${ }^{*} \mathrm{P}<0.05$, the incidence rate of ALA-PDT group obviously elevated with statistical significance compared with other two groups

$\mathbf{\Delta} P>0.05$, the incidence rates of OFI-ALA-PDT group and OFI group had no significant differences through comparison
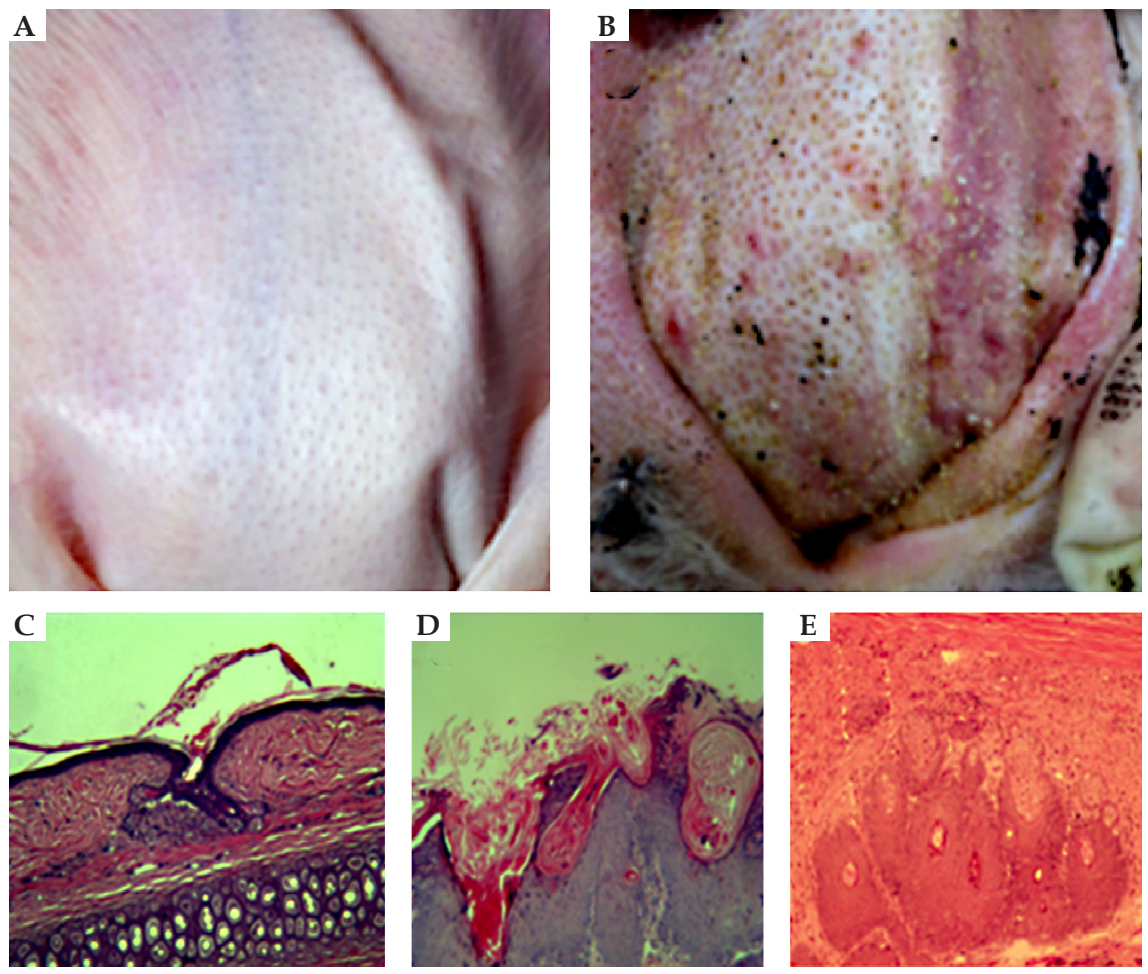

Figure 1:

Establishment of the rabbit ear model of acne. The normal ear (a) and acne model ear (b) are recorded and the histological observation reflected the changes in the sebaceous gland and hair follicle between the normal control (c) and acne model (d, e) (H\&E x100)

\section{TABLE 2: The comparison of effectiveness rate of the four groups 14 days after treatment}

\begin{tabular}{lllllll} 
Group & No. of ears & Cure & Excellent & Good & Poor & Effectiveness rate (\%) \\
\hline OFI-ALA-PDT & 10 & 2 & 8 & 0 & 0 & $100^{*}$ \\
ALA-PDT & 10 & 2 & 4 & 2 & 2 & $60^{* \Delta}$ \\
OFI & 10 & 0 & 4 & 6 & 0 & $40^{* \Delta}$ \\
Blank control group & 10 & 0 & 2 & 4 & 4 & $20^{* \Delta}$
\end{tabular}

${ }^{*} \mathrm{P}<0.05$, the effectiveness rate of OFI-ALA-PDT group obviously elevated with statistical significance compared with other three groups.

$\Delta \mathrm{P}>0.05$, the effectiveness rates of ALA-PDT group, OFI group and blank control group had no significant differences through multiple comparison. 


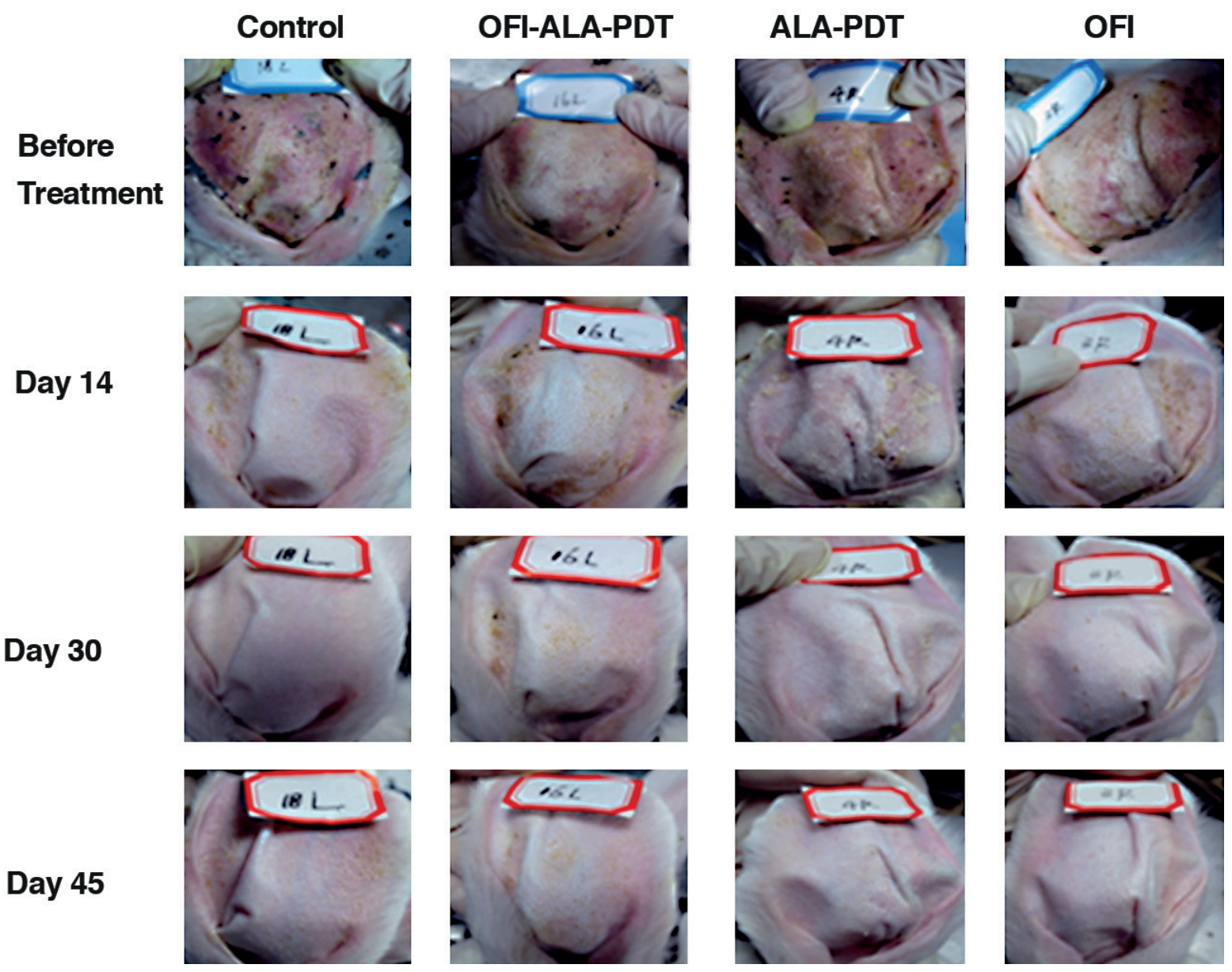

FIGURE 2: Treatment of rabbit ear model of acne in OFI-ALA-PDT, ALA-PDT, OFI and blank control group. The effects were recorded at days 14,30 and 45 after treatment
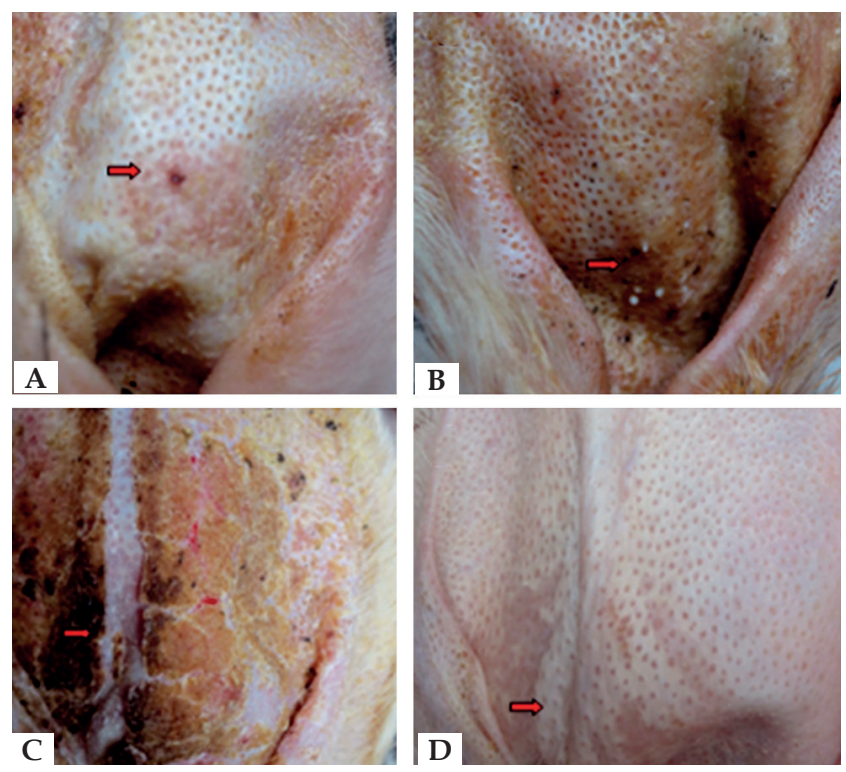

FigURE 3: Adverse events were observed in group OFI-ALA-PDT (a), ALA-PDT (b, c, d)
A large number of studies published have confirmed that ALA-PDT by red light is a highly effective treatment for acne. ${ }^{16-20}$ Along with ALA-PDT carried out extensively, more and more adverse events have been reported, such as pain, burning sensation, itching, pricking, edema, erythema, pustules, acneiform eruption, exfoliative or secondary pigmentation. A trend towards more serious adverse events was observed with increasing ALA concentration. Hence, promotion of traditional ALA-PDT was restricted by its adverse events.

In order to reduce the adverse events of ALA-PDT, investigators conducted different researches. Conventional methods include cold spray, cold compress, use of repair mask or sunscreen cream after photodynamic therapy, but these methods only have limited role. Nowadays, photosensitizer is a hot topic. M.S. Jang et al. introduced new photosensitizers (indocyanine green [ICG] and indole-3-acetic acid [IAA]) in photodynamic therapy for acne. This study made a good effect, and only produced slight erythema (6/34) and pruritus (8/34) after each irradiation, which was relieved within a few hours. ${ }^{21}$

PDT requires three essential components: a photosensitizer, a light source, and oxygen. ${ }^{22}$ Nowadays, the researches for the 
purpose of reducing the adverse events of ALA-PDT focus on the change of photosensitizer and irradiation dose, but there's no research on the improvement of the irradiation pathway. According to the optical transmission model theory in human tissue, the amount of light absorption in the skin tissue raised with the increase of absorption coefficient of each skin layer. Absorption coefficient of epidermis is much larger than layers of the dermis and subcutaneous tissue. ${ }^{23}$ The main barrier to the absorption of photosensitizers is the stratum corneum. Therefore, in traditional external ALA-PDT, the optical energy is mostly absorbed by the epidermis, instead, the sebaceous glands that should be treated only absorb reduced energy. So, patients can not only experience serious adverse events but also acquire decreased effectiveness. On the basis of the theory, we want to optimize the program on PDT for acne through improving radiation mode.

Scholars in South Korea used photodynamic therapy with intra-lesional injection of ALA to treat human acne, this method decreased the related adverse events. ${ }^{24}$ This research inspired us that meticulous treatment targets to the local inflammatory papules is efficient. In our study, intra-lesional irradiation was achieved by optical fiber, which guaranteed enough energy to sebaceous glands but epidermis only absorbed little energy. The modified ALA-PDT reduced adverse reaction, achieved high efficacy and maximized advantage of traditional ALA-PDT. But it did not enhance longterm effect of traditional ALA-PDT. OFI-ALA-PDT group finally achieved similar efficacy with traditional ALA-PDT group, and the efficiency was significantly higher in the early time. OFI-ALA-PDT showed a statistical superiority in effect at 14 days after treatment compared with conventional ALA-PDT $(P<0.05)$. This illustrated that this modified ALA-PDT therapy in the treatment of rabbit ear acne model is effective or at least is not inferior to traditional ALAPDT. Whereas the symptoms of discomfort and adverse events rates in OFI-ALA-PDT group were significantly lower than ALA-PDT group, indicating that the modified ALA-PDT has higher safety compared with traditional ALA-PDT.

However, this study also has some limitations. On the one hand, animal model induction of acne is temporary, which may have the limited efficacy assessment among the different treatment groups. On the other hand, the present study design was unblinded and the assessment was made with knowledge of the treatment groups, which can also cause bias. Therefore, future blinded studies using persistent animal model of acne are needed to clarify it further.

\section{CONCLUSION}

Here we showed the intra-tissue OFI-ALA-PDT treatment had no improved efficacy on treating rabbit ear acne model but had higher safety and better tolerability and our results might provide reference for the use of ALA-PDT in clinic acne therapy.

\section{ACKNOWLEDGEMENT}

This study was supported by the Health and Family Planning Commission of Sichuan Province (150222). 


\section{REFERENCES}

1. van de Kerkhof PC, Kleinpenning MM, de Jong EM, Gerritsen MJ, van DoorenGreebe RJ, Alkemade HA. Current and future treatment options for acne. J Dermatolog Treat. 2006;17:198-204.

2. Ma L, Xiang LH, Yu B, Yin R, Chen L, Wu Y, et al. Low-dose topical 5-aminolevulinic acid photodynamic therapy in the treatment of different severity of acne vulgaris. Photodiagnosis Photodyn Ther. 2013;10:583-90.

3. Ding HL, Wang XL, Wang HW, Huang Z. Successful treatment of refractory facial acne using repeat short-cycle ALA-PDT: Case study. Photodiagnosis Photodyn Ther. 2011;8:343-6.

4. Wang XL, Wang HW, Zhang LL, Guo MX, Huang Z. Topical ALA PDT for the treatment of severe acne vulgaris. Photodiagnosis Photodyn Ther. 2010;7:33-8.

5. Hongcharu W, Taylor CR, Chang Y, Aghassi D, Suthamjariya K, Anderson RR. Topical ALA-photodynamic therapy for the treatment of acne vulgaris. J Invest Dermatol. 2000;115:183-92.

6. Ko DY, Kim KH, Song KH. Comparative Study of Photodynamic Therapy with Topical Methyl Aminolevulinate versus 5-Aminolevulinic Acid for Facial Actinic Keratosis with Long-Term Follow-Up. Ann Dermatol. 2014;26:321-31.

7. Yin R, Lin L, Xiao Y, Hao F, Hamblin MR. Combination ALA-PDT and ablative fractional Er:YAG laser $(2,940 \mathrm{~nm})$ on the treatment of severe acne. Lasers Surg Med. 2014;46:165-72.

8. Chaves YN, Torezan LA, Niwa AB, Sanches Junior JA, Festa Neto C. Pain in photodynamic therapy: mechanism of action and management strategies. An Bras Dermatol. 2012;87:521-6

9. Kligman AM, Katz AG. Pathogenesis of acne vulgaris. I. Comedogenic properties of human sebum in external ear canal of the rabbit. Arch Dermatol. 1968 :98:53-7.

10. Kligman AM, Kwong T. An improved rabbit ear model for assessing comedogenic substances. Br J Dermatol. 1979;100:699-702.

11. Ma L, Xiang LH, Yu B, Yin R, Chen L, Wu Y, et al. Low-dose topical 5-aminolevulinic acid photodynamic therapy in the treatment of different severity of acne vulgaris. Photodiagnosis Photodyn Ther. 2013;10:583-90.

12. Cosgarea R, Susan M, Crisan M, Senila S. Photodynamic therapy using topical 5-aminolaevulinic acid vs. surgery for basal cell carcinoma. J Eur Acad Dermatol Venereol. 2013;27:980-4.

13. Kang HK, Yun JH, Son YM, Roh JY, Lee JR. Photodynamic Therapy for Bowen's Disease of the Vulva Area. Ann Dermatol. 2014;26:241-5.

14. Qian G. Wang S, Deng D, Yang G. Is the step-up therapy of topical 5-aminolevulinic acid photodynamic therapy effective and safe for the patients with recalcitrant facial flat wart? Dermatol Ther. 2014;27:83-8.

15. Sotiriou E, Panagiotidou D, loannides D. 5-Aminolevulininic acid photodynamic therapy treatment for tinea cruris caused by Trichophyton rubrum: report of 10 cases. J Eur Acad Dermatol Venereol. 2009;23:341-2.

16. Yang GL, Zhao M, Wang JM, He CF, Luo Y, Liu HY, et al. Short-term clinical effects of photodynamic therapy with topical 5 -aminolevulinic acid for facial acne conglobata: an open, prospective, parallel-arm trial. Photodermatol Photoimmunol Photomed. 2013 0ct;29:233-8.

17. Wang HW, Lv T, Zhang LL, Guo MX, Stepp H, Yang K, et al. Prospective study of topical 5 -aminolevulinic acid photodynamic therapy for the treatment of moderate to severe acne vulgaris in Chinese patients. J Cutan Med Surg. 2012;16:324-33.

18. Jeong E, Hong JW, Min JA, Lee DW, Sohn MY, Lee WJ, et al. Topical ALAPhotodynamic Therapy for Acne Can Induce Apoptosis of Sebocytes and Downregulate Their TLR-2 and TLR-4 Expression. Ann Dermatol. 2011;23:23-32.

19. Fabbrocini G, Cacciapuoti S, De Vita V, Fardella N, Pastore F, Monfrecola G. The effect of aminolevulinic acid photodynamic therapy on microcomedones and macrocomedones. Dermatology. 2009;219:322-8.

20. Oh SH, Ryu DJ, Han EC, Lee KH, Lee JH. A comparative study of topical 5-aminolevulinic acid incubation times in photodynamic therapy with intense pulsed light for the treatment of inflammatory acne. Dermatol Surg. 2009;35:1918-26.

21. Jang MS, Doh KS, Kang JS, Jeon YS, Suh KS, Kim ST. A comparative split-face study of photodynamic therapy with indocyanine green and indole-3-acetic acid for the treatment of acne vulgaris. Br J Dermatol. 2011;165:1095-100.

22. Wan MT, Lin JY. Current evidence and applications of photodynamic therapy in dermatology. Clin Cosmet Investig Dermatol. 2014;7:145-63.

23. Jacques SL. Optical properties of biological tissues: a review. Phys Med Biol. 2013;58:R37-61

24. Ryou JH, Lee SJ, Park YM, Kim HO, Kim HS. Acne-photodynamic therapy with intra-lesional injection of 5-aminolevulinic acid. Photodermatol Photoimmunol Photomed. 2009;25:57-8.

\section{MAILING ADDRESS:}

Wei Liu

Sichuan Academy of Medical Sciences E Sichuan Provincial

People's Hospital

No. 32, Western 2nd Section

1st Ring Road,

Chengdu City,

610072 Sichuan Province, China.

Email: lwlucky622@163.com

How to cite this article: Wang Q, Jiang C, Liu W, Chen J, Lin X, Huang X, Duan X. A new optical intra-tissue fiber irradiation ALA-PDT in the treatment of acne vulgaris in rabbit model: improved safety and tolerance. An Bras Dermatol. 2017;92(3):351-5. 